\title{
Serum and Urine Levels of Magnesium in Adult Males with Type 2 Diabetes Mellitus in Jeddah,
} Saudi Arabia

Syed M. FARID ${ }^{1}$

${ }^{1}$ Department of Nuclear Engineering, King Abdulaziz University, Jeddah, Saudi Arabia.

\section{ABSTRACT}

The present study aimed to investigate the association of serum and urine magnesium $(\mathrm{Mg})$ levels of the patients living in Jeddah, Saudi Arabia, with type 2 diabetes mellitus (T2 DM) without complications. The effect of Mg deficiency on the prevalence of DM and its related complications has received great attention. Serum and urine Mg, fasting serum glucose (FSG), glycated hemoglobin (HbAlc), and urine creatinine (Creat) levels were determined in 68 diabetic subjects and 62 age-matched nondiabetic subjects (controls) using auto-analyzer and atomic absorption spectrometer. Body mass indices (BMI) of the subjects were also determined. The serum $\mathrm{Mg}$ levels in the patients with T2 DM were significantly lower than that in controls. BMI and the levels of FSG, HbAlc, and urine Mg were significantly higher and urine Creat level lower in diabetic subjects compared with nondiabetic subjects. Significantly lower serum Mg and significantly higher urinary Mg levels were observed in diabetic subjects with poor glycemic control (HbA Ic >7\%) compared with the nondiabetic population. The present correlation study revealed a highly significant correlation between FSG and HbA Ic $(r=0.846, P=0.000)$. Aging and increasing duration of DM alter the metabolism of $M g$ by decreasing its serum concentration and increasing its urinary excretion. This study also showed a significant negative association between serum Mg and FSG $(r=-0.408, P=0.039$ and HbAlc $(r=-0.478, P=0.043)$. Also, a significant negative association was observed between serum $\mathrm{Mg}$ and age of both patients with $T 2 \mathrm{DM}(r=-0.787, P=0.044)$ and controls $(r=-0.798, P=$ 0.041). Again, a significant negative correlation ( $r=-0.452, P=0.018$ ) was observed between serum $M g$ and urine $M g$ levels of the diabetic population of the study. DM and poor glycemic control alter the metabolism of Mg by increasing its urinary excretion and lowering its serum levels. Its clinical implications were discussed in this study.

Key words: Diabetes and glycemic control, magnesium, serum, urine

\section{INTRODUCTION}

Diabetes mellitus (DM) is a growing public health concern across the world, particularly in developing countries. The prevalence of type 2 DM (T2 DM) is rising much more rapidly because of increasing obesity and reduced activity levels as countries become more industrialized. This is true in most countries, and 6 of the top 10 countries with the highest rates are in Asia (1). The prevalence of DM in Saudi Arabia is at an alarming level. More than $25 \%$ of the adult population is suffering from DM, and this figure is expected to be more than double by 2030 (2). Half of the people more than 30 years of age are prone to DM. Saudi Arabia ranks seventh worldwide and the first in the gulf in terms of DM prevalence rates (3). T2 DM is the major problem and accounts for more than $90 \%$ of the cases. It is reported that around $9 \%$ of patients with DM die annually. Beyond the devastating humanitarian costs, DM threatens to subvert the gains of economic development globally as a consequence of spiraling costs of medical care (4).

Glycated hemoglobin (HbA1c) is considered a good method of assessing glycemic control. The higher the percentage of circulating $\mathrm{HbA1C}$ in DM, the poorer the mean diabetic control. Achieving near-normal HbA1c levels has been shown to reduce long-term complications. The HbA1c assay is recommended to determine whether treatment is adequate and to guide adjustments $(5,6)$. Accumulating evidence indicates that the metabolism of several trace elements is altered in DM. Deficiency and efficiency of some essential trace elements may play a role in the development of DM (7). Magnesium (Mg) 
is known to play an important role in carbohydrate metabolism. Its imbalance has been implicated in DM, both dietary Mg and low serum $\mathrm{Mg}$ levels. These are recently implicated as risk factors for hypertension, DM, and coronary artery disease. Hypomagnesemia occurs frequently in patients with DM, especially those with poor glycemic control (8-12).

Although some limitations may apply, serum $\mathrm{Mg}$ concentration is still used as the standard for evaluating Mg status in patients. Another approach for assessing Mg status is urinary Mg excretion. The results provide etiological information: while a high urinary excretion indicates renal wasting of $\mathrm{Mg}$, a low value suggests an inadequate intake or absorption $(10,13,14)$.

This study aimed to determine the serum and urine levels of $\mathrm{Mg}$ in diabetic and nondiabetic subjects with different states of glycemic control to know the status of this element and explore any association of FSG and $\mathrm{HbA1c}$ with $\mathrm{Mg}$ concentrations.

\section{MATERIALS AND METHODS}

\section{Selection of subjects}

This study was conducted among 68 male subjects with T2 DM (DM group) and 62 age-matched, nondiabetic male subjects (control group) within age range 35-65 years. All of them worked at the King Abdulaziz University. The patients attended the clinic of the University regularly. They were all urban residents of Jeddah city, Saudi Arabia. Before the start of the study, all control subjects and patients with DM were informed about the aim of the study via the distributed questionnaire, and all agreed to participate and signed the form. The study protocol was approved by the Research Ethics Committee of the University.

The inclusion and exclusion criteria were the same as those mentioned in previous reports $(15,16)$.

Sixty-two apparently healthy, nondiabetic subjects of similar socioeconomic status, who had routine medical checkups in the clinic, were recruited as control. No clinical or laboratory disorders were observed in the control group. Body weight and height were measured and used to calculate the body mass index (BMI), which was used as a measure of relative body weight. Following enrollment, both patients and controls were instructed not to change their lifestyle or their dietary habits and not to take any dietary supplements. The diet was not monitored.

Sample collection and preparation

Fasting blood samples were collected into labeled centrifuge tubes from the subjects by venipuncture after an 8- to 12-h overnight fast. The blood samples were centrifuged at 2000 rpm for 10 min using a desktop centrifuge, and the serum was separated and kept in labeled sample bottles at $-70^{\circ} \mathrm{C}$ until further analysis.

Serum urea and creatinine levels were also assayed to test for renal function. Fasting spot urine samples were also collected into sterile chemically clean universal containers for urine $\mathrm{Mg}$ determination and also for urine creatinine estimation to correct for urine flow rate of individuals. A certain amount of urine samples was taken into a metal-free glass tube and mixed with concentrated nitric acid to remove interfering of organic compounds. The resulting solution was then centrifuged.

\section{Instrumentation}

The sera were analyzed for HbA1c and fasting serum glucose (FSG) using an auto-analyzer (Roche Modular P-800, Germany). The trace concentrations of $\mathrm{Mg}$ in serum and urine samples were measured using the Graphite Furnace Atomic Absorption Spectrometer (Varian, Model Spectra AA 30P, Australia) by a calibration method. The accuracy of determination was evaluated by measuring the metal contents of certified biological reference materials (Seronorm Trace Elements Serum; Nycomed Pharma, Oslo, Norway) $(15,16)$.

\section{Statistical analysis}

The results were presented as mean \pm standard deviation (SD). The significance of difference between the groups was tested using the t test. Association between variables was determined using the Pearson's correlation analysis on Microsoft Excel and SPSS software version 16 (SPSS, IL, USA). Statistical significance was determined at a $P$ value less than 0.05 .

\section{RESULTS}

A total of 130 male subjects were included in this study; 68 of them were patients with T2 DM and 62 age-matched nondiabetic healthy control subjects. The patients and controls were aged between 35 and 65 years. BMI was calculated using the standard formula (weight in kilograms/height in square meters, $\left.\mathrm{kg} / \mathrm{m}^{2}\right)$. General characteristics and laboratory findings of the population studied are shown in Table 1. The mean age \pm SD of the patients and control subjects was $60.08 \pm 6.29$ and $58.05 \pm 5.88$ years, respectively. The patients with DM were generally heavier than the control subjects. The BMI of patients with DM was also found to be more compared with that of the controls (Table 1). The results of the BMI indicated that 
TABLE 1: Descriptive data and laboratory findings of diabetic and nondiabetic subjects.

\begin{tabular}{lccc}
\hline Data & $\begin{array}{c}\text { Diabetic subjects } \\
(n=68)\end{array}$ & $\begin{array}{c}\text { Nondiabetic subjects } \\
(n=62)\end{array}$ & $P$ value \\
\hline Sex & Male & Male & 0.038 \\
Age (year) & $60.08 \pm 6.29$ & $58.05 \pm 5.88$ & 0.018 \\
Duration of diabetes (year) & $5.73 \pm 3.06$ & & 0.001 \\
BMI (kg/m²) & $28.96 \pm 1.86$ & $24.58 \pm 1.52$ & 0.004 \\
Fasting serum glucose (mmol/L* & $10.16 \pm 3.59$ & $4.36 \pm 0.21$ & 0.005 \\
HbA1c $(\%) *$ & $9.34 \pm 2.16$ & $5.12 \pm 1.07$ & 0.006 \\
Creatinine (mg/dL)* & $0.98 \pm 0.25$ & $0.90 \pm 0.24$ & 0.003 \\
Urine creatinine (mg/dL)* & $117.63 \pm 60.21$ & $181.11 \pm 56.52$ & 0.043 \\
Serum Mg (mg/L)* & $14.10 \pm 3.89$ & $5.78 \pm 3.62$ & \\
Urine Mg (mg/g Creat)* & $11.67 \pm 5.49$ & & \\
\hline *Significant difference in the mean. & & & \\
\end{tabular}

the diabetic subjects were overweight. A significant difference in the BMI of the patients with DM was found compared with the control group (Table 1). The FSG, HbA1c were significantly higher in diabetic than in nondiabetic subjects (Table 1). Urine creatinine was significantly higher in nondiabetic than in diabetic subjects (Table 1).

A significant decrease in serum Mg was noted in diabetic subjects compared with controls (Table 1). The urinary Mg levels were found to be significantly higher in the diabetic compared with the control population (Table 1).

Table 2 shows the serum and urine Mg levels in diabetic subjects with poor glycemic control ( $\mathrm{HbATC}>7 \%)$ and nondiabetic subjects. The serum Mg was significantly lower while urine $\mathrm{Mg}$ levels were significantly higher in diabetic subjects with poor glycemic controls than in nondiabetic controls (Table 2).

The present study also showed a significant negative association between serum $\mathrm{Mg}$ and age of both patients with $\mathrm{DM}$ and controls $(r=-0.787, P=0.044 ; r=-0.798, P=0.041)$.
The results showed that the serum Mg levels decreased significantly $(r=-0.267, P=0.037)$ and urinary excretion increased $(r=0.385, P=0.015)$ with the duration of DM.

This correlation study revealed a highly significant correlation between FSG and HbA1c $(r=0.846, P=0.000)$ for patients with DM. Moreover, a significantly negative correlation was observed between serum Mg and FSG $(r=-0.408, P=0.039)$ (Fig. 1) and HbA1C ( $r=-0.478, P=0.043)$ (Fig. 2) for patients with DM. A significant positive correlation $(r=0.356, P=$ 0.013) was observed between HbA1c and urinary loss of $\mathrm{Mg}$ in patients with T2 DM (Fig. 3). A significant negative correlation ( $r$ $=-0.452, P=0.018$ ) was observed between serum and urine Mg levels of patients with DM (Fig. 4).

\section{DISCUSSION}

Accumulating evidence indicates that the metabolism of several trace elements is altered in DM (8). Mg is known to play an important role in carbohydrate metabolism. Its imbalance has

TABLE 2: Serum and urine Mg levels in different states of glycemic control in diabetic and nondiabetic subjects.

\begin{tabular}{|c|c|c|}
\hline Control state & Serum Mg (mg/L) & Urine Mg (mg/g Creat) \\
\hline Good $\mathrm{HbA}_{1 \mathrm{c}}(<7 \%) \quad(n=13)$ & $18.60 \pm 4.57$ & $10.74 \pm 4.99$ \\
\hline Poor $\mathrm{HbA}_{1 \mathrm{c}}(>7 \%)(n=55)$ & $13.81 \pm 3.96$ & $11.93 \pm 6.98$ \\
\hline$P$ value & 0.039 & 0.055 \\
\hline Poor $\mathrm{HbA}_{1 \mathrm{c}}(>7 \%)(n=55)$ & $13.81 \pm 3.96$ & $11.93 \pm 6.98$ \\
\hline Nondiabetic subjects $(n=62)$ & $18.51 \pm 4.86$ & $5.78 \pm 3.62$ \\
\hline$P$ value & 0.038 & 0.044 \\
\hline
\end{tabular}




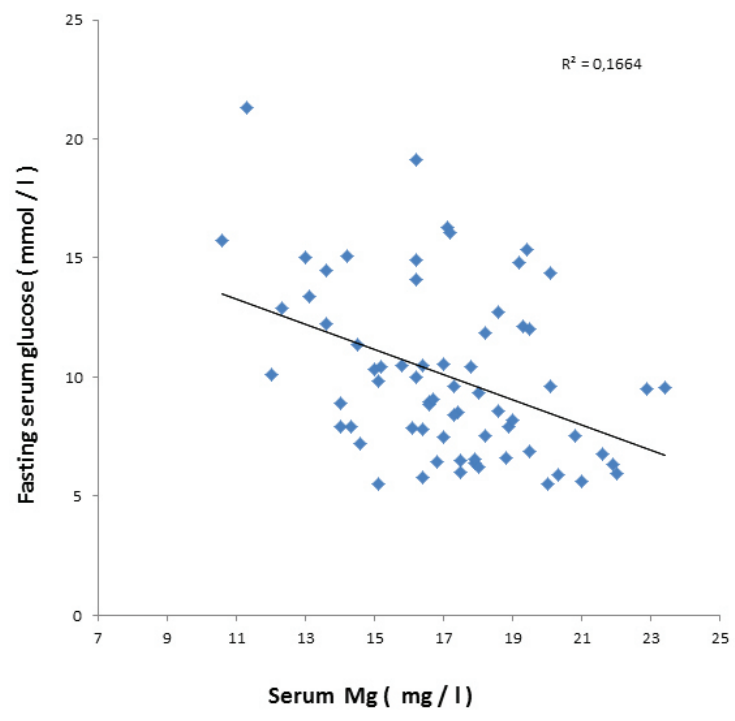

FIGURE 1: Correlation between serum Mg and FSG of patients with DM.

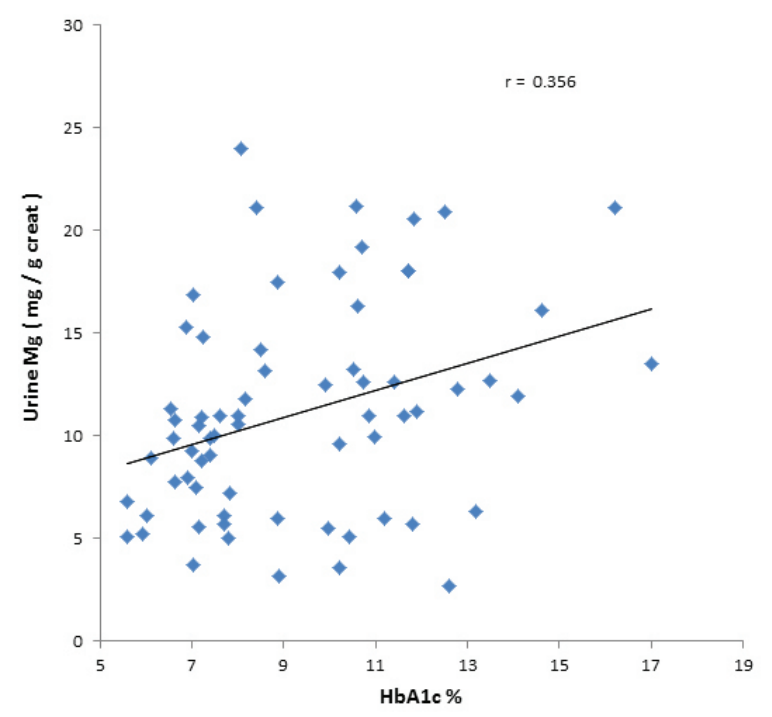

FIGURE 3: Correlation graph of HbA1c and urine Mg of patients with DM.

been implicated in DM, as a cause and a consequence $(6,14)$. The interest in the important roles played by $\mathrm{Mg}$ in various cell processes in the body has increased recently $(11,17)$. Studies have shown that Mg levels are lower in patients with DM compared with nondiabetic controls. The association of hypomagnesemia with poor glycemic control and various long-term complications of DM has been reported (17-20). The serum and urine levels of $\mathrm{Mg}$ in diabetic subjects and in different states of glycemic control, as determined by HbA1c levels, were determined in this study. The possible correlation of age and duration of DM with serum and urine levels of $\mathrm{Mg}$ was also examined.

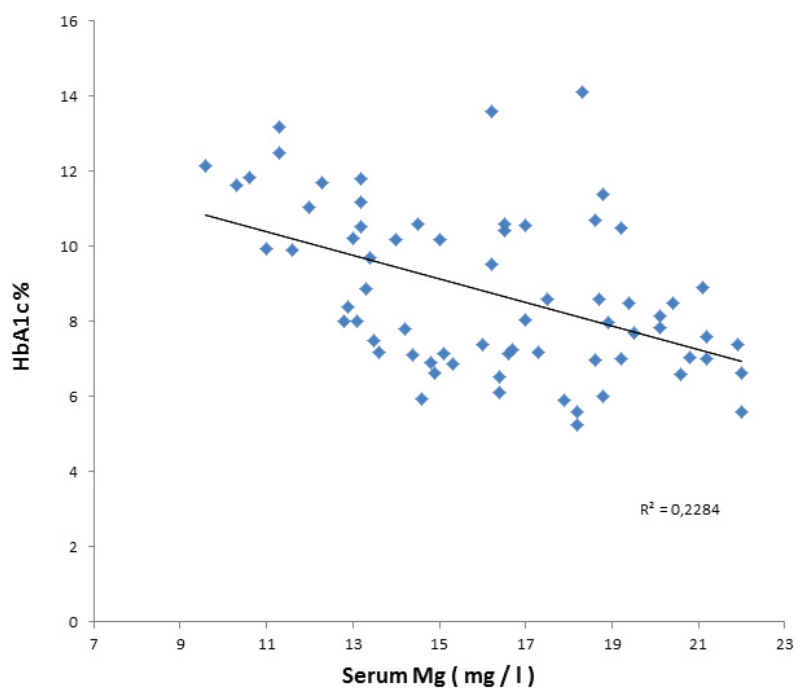

FIGURE 2: Correlation between serum Mg and $\mathrm{HbA1c}$ of patients with DM.

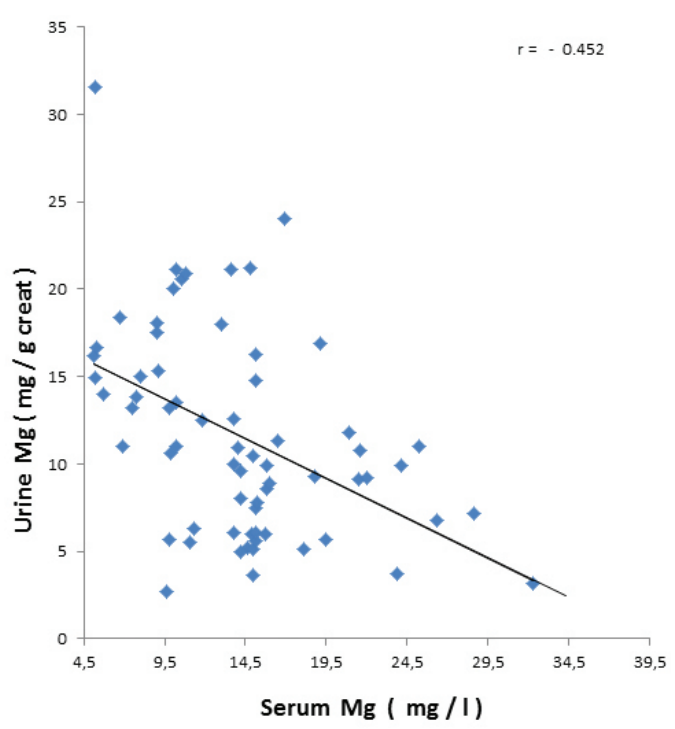

FIgURE 4: Correlation between serum Mg and urine Mg of patients with DM.

The BMI, FSG, and HbA1c were significantly higher in the diabetic population compared with the nondiabetic population of the study. The higher values of BMI, FSG, and HbA1c recorded for patients with DM in this study were consistent with previous reports (21-25). This study demonstrated a good agreement between FSG and HbA1c in the assessment of glucose metabolism in DM. A significant positive correlation was found in both patients with DM and control subjects ( $r=0.846$ and 0.878 , respectively, $P=0.000)$, which was consistent with the findings of other studies $(22,24,25)$. The main use of HbA1c is to assess longterm glycemic control in managing patients with DM, provide 
valuable information about the average blood glucose levels in the previous 1-2 months, and monitor compliance of patients to treatment regimens (21,23-26). Apart from classical risk factors such as dyslipidemia, elevated HbA1c has now been regarded as an independent risk factor for cardiovascular disease (CVD) in subjects with or without DM. The estimated risk of CVD has been shown to be increased by $18 \%$ for each $1 \%$ increase in the absolute HbA1c value in the diabetic population (21,22, 25-27).

The result of the present study revealed a significant lowering of serum Mg level in T2 DM (Table 1) compared with healthy controls, which was consistent with the findings of other studies $(6,27-29)$. The present study results demonstrated some degree of variability in the serum and urine concentrations of $\mathrm{Mg}$ with age and duration of DM. A significant inverse correlation of serum Mg with age in both patients with DM and normal controls was observed, which was similar to the findings of previous studies $(24,30-32,37)$. A number of surveys have shown Mg, zinc (Zn), selenium (Se), and chromium intakes by old persons to be lower than the corresponding nutrient intakes; this may be attributed to changes in mineral bioavailability with aging. Aging has been previously associated with low intracellular Mg concentration, which is probably the consequence of insulin resistance due to aging (30, 33-35).

This study showed a significant negative correlation of serum $\mathrm{Mg}$ with FSG and HbA1c. The results demonstrated that serum $\mathrm{Mg}$ concentrations were dependent on the degree of glucose control as determined by correlation analysis between $\mathrm{HbA1C}$ and $\mathrm{Mg}$ concentrations. The findings suggested that the patients with poorly controlled DM had a decreased level of Mg, potentiating the risk for DM-related complications. The association of hypomagnesemia with poor glycemic control and various longterm complications of DM has been reported $(10,29,36,37,38)$. $\mathrm{Mg}$ deficiency has also been argued as a probable causal factor of dyslipidemia, which constitutes an alert for the chronic complications of DM, such as atherosclerosis $(6,29,37,39)$.

Urine concentrations of $\mathrm{Mg}$ were observed to be significantly higher in patients with DM than in the nondiabetic population in the present study. El-Yazigi et al. (40) made similar observations on the effects of the diabetic state and related disorders on the urinary excretion of $\mathrm{Mg}$ in patients, where higher urinary excretion of this element was demonstrated in the diabetic state. Different authors also reported increased urinary $\mathrm{Mg}$ loss in diabetic subjects $(22,29,41)$. This study also observed decreased serum $\mathrm{Mg}$ and increased urinary $\mathrm{Mg}$ loss with increasing age and duration of DM in the diabetic population. Hypermagnesuria in diabetic subjects has been attributed to osmotic diuresis. Glycosuria, which accompanies the diabetic state, impairs renal tubular reabsorption of $\mathrm{Mg}$ from the glomerular filtrate. An increased urinary loss of $\mathrm{Mg}$ may be attributed to reductions in renal function with aging and duration of DM $(30,38)$. Low Mg, $\mathrm{Se}$, and $\mathrm{Zn}$ status has also been reported in the elderly population $(30,43)$.

Hypomagnesemia is common among patients with DM, especially those with poor metabolic control $(6,34)$. Polyuria caused by hyperglycemia, coupled with hyperinsulinemia, tended to increase renal excretion of $\mathrm{Mg}$ or decrease renal reabsorption of $\mathrm{Mg}$, thereby resulting in hypomagnesemia in $\mathrm{T} 2$ DM. An inadequate intake of dietary Mg in patients with diabetes may also cause hypomagnesemia. Hypomagnesemia occurs at an incidence of $13.5 \%-47.7 \%$ among patients with T2 DM. The increased incidence of hypomagnesemia among patients with $\mathrm{T} 2$ DM presumably is multifactorial $(10,21,28,39)$.

Clinically, hypomagnesemia may be defined as a serum $\mathrm{Mg}$ concentration $\leq 1.6 \mathrm{mg} / \mathrm{dL}$ or $>2$ SD below the mean of the general population $(10,29,32)$. The present study demonstrated that $38 \%$ of the patients with DM had significanthypomagnesemia with the serum $\mathrm{Mg}$ level less than $1.6 \mathrm{mg} / \mathrm{dL}$. Studies have reported incidence rates of $13.5 \%-47.7 \%$ in diabetic subjects $(10,28,29,32)$. Jeddah has a hot and humid climate year-round. Summer temperatures are very hot, often crossing the $430 \mathrm{C}$ $(1090 \mathrm{~F})$ mark in the afternoon and dropping to $300 \mathrm{C}(860 \mathrm{~F})$ in the evening. The highest temperature ever recorded in Jeddah was $52^{\circ} \mathrm{C}\left(125.6^{\circ} \mathrm{F}\right)$. The average temperature in Jeddah is $28.4^{\circ} \mathrm{C}\left(83^{\circ} \mathrm{F}\right)$. The high temperature in Jeddah (especially in summer, April to November) might also be a contributing factor to the higher incidence of hypomagnesemia in the present study. A possible correlation between Mg deficiency and climate variations is speculated, contributing to the increase in deaths due to heart disease and DM. High temperatures would increase sweat losses. Consequently, among the minerals, Mg would be the most affected because the losses would not be compensated by the diet and water intake, thereby increasing the risk of these diseases $(6,39,44)$.

Duration of DM also seems to exert a significant effect on the serum and urine $\mathrm{Mg}$ concentration of the diabetic population studied. Serum $\mathrm{Mg}$ levels decreased with the increasing duration of, and their urinary excretion increased with the increasing duration of DM. The decreased serum levels may be attributed 
to the increased urinary loss of this trace element. Reduction in renal functions with the increasing duration of DM has been implicated in the urinary loss of trace elements $(38,45)$. The strong correlation of $\mathrm{Mg}$ with duration of disease suggests that elderly diabetic patients are more susceptible to develop $\mathrm{Mg}$ deficiency $(30,37)$.

Higher urine $\mathrm{Mg}$ concentrations and lower serum $\mathrm{Mg}$ concentrations were seen in diabetic subjects with poor glycemic control compared with nondiabetic controls. Increased urinary excretion of $\mathrm{Mg}$ in poor glycemic control has also been attributed to hyperglycemia, glucosuria, and osmotic diuresis $(38,46)$. Serum $\mathrm{Mg}$ correlated negatively with urine $\mathrm{Mg}$ in the diabetic population of this study. The status of body $\mathrm{Mg}$ balance is determined by the renal excretion. Thus, when the $\mathrm{Mg}$ status is suboptimal, $\mathrm{Mg}$ receptors on the thick ascending limb of the loop of Henle sense the need for $\mathrm{Mg}$ retention and cause more reabsorption (38). Hence, the lower the urine excretion of $\mathrm{Mg}$, the higher the serum $\mathrm{Mg}$ concentration and vice versa. Therefore, it is concluded that DM and poor glycemic control alter the metabolism of $\mathrm{Mg}$ by causing hypermagnesuria and hypomagnesemia.

Deficiencies of $\mathrm{Mg}$ in diet and serum have been associated with an increased risk of developing glucose intolerance and DM. However, increased $\mathrm{Mg}$ intake is associated with a significant decline in the incidence of T2 DM $(28,32,39,47,48)$. Thus, foods rich in $\mathrm{Mg}$ (such as nuts, green vegetables, soybeans, and whole grains) may provide protection against this chronic disease. It is suggested that patients with DM should take Mg-rich foods every day, which provides recommended intakes of $\mathrm{Mg}$ and maintains normal storage levels of this mineral. Dietary intake of $\mathrm{Mg}$ can often restore mildly depleted $\mathrm{Mg}$ levels, but Mg supplementation is required to restore very low $\mathrm{Mg}$ levels to normal.

$\mathrm{Mg}$ is involved in nearly every physiological system. Although $\mathrm{Mg}$ supplementation is used for treating some cardiovascular disorders, the beneficial effects of $\mathrm{Mg}$ replacement in preventing DM-related complications have not yet been proven in longterm studies. Some observations have suggested that chronic Mg supplementation may be useful in treating patients with DM, improving the glycemic control and preventing the development of chronic complications $(49,50)$. Further studies are needed to define the clinical scope of therapy with $\mathrm{Mg}$.

\section{CONCLUSIONS}

The present study demonstrated a significant correlation of $\mathrm{HbA1C}$ with the FSG levels. HbA1c can be used as a reliable, feasible, and fairly accurate tool for screening DM. The findings of this study indicated that diabetic subjects are Mg deficient and the diabetic state enhances the urinary loss of this element. It also found that hypomagnesemia was associated with increased levels of FSG and $\mathrm{HbA1c}$. In diabetic subjects, aging and increasing duration of DM enhance the urinary loss of $\mathrm{Mg}$. Therefore, it is concluded that DM and poor glycemic control alter the metabolism of $\mathrm{Mg}$ by causing hypermagnesuria and hypomagnesemia. It is prudent to monitor Mg levels in all patients with T2 DM regularly and treat the condition whenever possible. All persons with T2 DM must be started on primary prevention by encouraging healthy lifestyle diets so as to reduce the risk of coronary heart disease and atherosclerosis. It is also suggested that patients with DM should take Mg-rich foods such as whole grains, legumes, fruits, and vegetables (especially dark green, leafy vegetables) every day, which provide recommended intakes of $\mathrm{Mg}$ and maintain storage levels of this mineral. Dietary intake of $\mathrm{Mg}$ can often restore mildly depleted $\mathrm{Mg}$ levels, but $\mathrm{Mg}$ supplementation is required to restore very low magnesium levels to normal. Thus, studies on the role of $\mathrm{Mg}$ supplementation in T2 DM in the Saudi population are recommended. Health care providers should invest more effort in diet changes rather than focusing on micronutrient supplementation to achieve metabolic control in their patients.

\section{REFERENCES}

1. Asmita PB, Jayashree GV. Glycosylated hemoglobin and fasting blood glucose levels in type 2 diabetes mellitus. Indian J Basic Appl Med Res 2014; 3(2):555-559.

2. Diabetes among Saudis, a major issue. November, 2012 (Arab News).

3. Diabetes: KSA is 7th. in the world, 1st. in Gulf, June, 2015 (Arab News).

4. IDF - International Diabetic Federation : 2010 - 2012 Strategy (www.idf.org/2010-2012-strategy).

5. Deepa R, Arvind K, Mohan V. Diabetes and risk factors for coronary artery disease. Curr Sci 2002;83(12):1497 - 1505.

6. Mane M, Chaudhari GR, Reddy EP. Hypomagnesemia in diabetic patients and biochemical action on the cardiovascular system. Int J Biol Med Res 2012;3(1):12731276.

7. Venkataraman K, Kao SL, Thai AC, Salim A, Lee JJM, Heng D, Tai ES, Khoo EY. Ethnicity modifies the relation between fasting plasma glucose and $\mathrm{HbA1c}$ in Indians, Malays and Chinese. Diabet Med. 2012;29 : 911-917.

8. Kazi TG, Afridi HI, Kazi N, Jamali MK, Arain MB, Jalbani N, Kandhro GA. Cu, Cr, Mn, Fe, Ni, and $\mathrm{Zn}$ levels in biological samples of diabetes mellitus patients. Biol Trace Elem Res 2008;122: 1-18.

9. Supriya SM, Roppa M, Venkata BKP, Raghavendra DS. Hypomagnesemia, lipid profile and glycosylated hemoglobin in type 2 diabetes mellitus patients. Int J Chem Pharma Res 2012; 1(5):116 - 123. 
10. Pham PC, Pham PM, Pham SV, Miller JM, Pham PT. Hypomagnesemia in patients with type 2 diabetes. Clin J Am Soc Nephrol 2007;2:366-373.

11. Tosiello L. Hypomagnesemia and diabetes mellitus. Arch Intern Med 1996;156:1143-1148.

12. Valk HW. Magnesium in diabetes mellitus. Netherlands J Med 1999; 54:139-146.

13. Lopez RR, Willett WC, Rimm EB, Liu S, Satampper MJ, Manson $\mathrm{JE}$ et al. Magnesium intake and risk of type 2 diabetes in men and women. Diabetes Care 2004;27(1):134-140.

14. Rasheed $H$, Elahi $S$, Ajaz $H$. Serum magnesium and atherogenic lipid fractions in type 2 diabetic patients of Lahore. Pak Biol Trace Elem Res 2012;148(2):165-169.

15. Farid SM. The association between serum glucose and serum lead and selected trace elements in type 2 diabetes mellitus patients in Jeddah, Saudi Arabia. Med J Islamic World Aca Sci 2012; 20(4):130-139.

16. Farid SM, Abulfaraj TG. Trace mineral status related to levels of glycated hemoglobin of type 2 diabetic subjects in Jeddah, Saudi Arabia. Med J IAS 2013; 21(2): 47-56.

17. Elin RJ. Magnesium metabolism in health and disease. Dis Mon. 1988; 34 : 161-218.

18. Paolisso $G$ and Barbagallo M. Hypertension, diabetes mellitus, and insulin resistance. The role of intracellular magnesium. Am J Hypertens 1997;10(3):346-355.

19. Mangou A, Grammatikopoulon MG, Mirkopoulon D, Sailer $\mathrm{N}$, Kotzamanidis G. Association between diet quality, health status and diabetic complications in patients with type 2 diabetes and comorbid obesity. Endocrinol Nutr 2012; 59:109-116.

20. Touyz RM. Magnesium in clinical medicine. Front Biosci 2004; 9:1278-1293.

21. Dechent WJ and Ketteler M. Magnesium basics. Clin Kidney J. 2012; 5:i3-i14.

22. Nsonwu AC, Usoro CAO, Etukudo MH, Usoro IN. Glycemic control and serum and urine levels of $\mathrm{Zn}$ and $\mathrm{Mg}$ in diabetics in Calabar, Nigeria. Pak J Nutr 2006 a; 5(1):75-78.

23. Praveeena $S$, Sujatha $P$, Sameera K. Trace elements in diabetes mellitus. J Clin Diag Res 2013;7(9):1863-1865.

24. Goud BKM, Nayal B, Devi OS, Sathisha TG, Shivashanker S, Devaki RN. Relation of calculated $\mathrm{HbAlc}$ with fasting plasma glucose and duration of diabetes. Int J Appl Biol Pharma Technol 2011;2(2):58-61.

25. Ramachandran A, Riddle MC, Kabali C, Gerstein HC. Relationship between $\mathrm{A} 1 \mathrm{C}$ and fasting plasma glucose in dysglycemia or type 2 diabetes. Diabetes Care 2012; 35(4): 749-753

26. Pasupathi P, Manivannan P, Uma M, Deepa M. Glycated hemoglobin ( $\mathrm{HbA1c}$ ) as a stable indicator of type 2 diabetes. Int J Pharm Biomed Res 2010;1(2):53-56.

27. Sikaris K. The correlation between hemoglobin A1c to blood glucose. J Diabetes Sci Teclnol 2009; 3(3):429-438.

28. Chaudhary DP, Sharma $R$, Bansal DD. Implications of magnesium deficiency in type 2 diabetes. Biol Trace Elem Res 2010;134; 119-129.

29. Dasgupta A, Sarma D, Saikia UK. Hypomagnesemia in type 2 diabetes mellitus. Indian J Endocr Metab 2013;16:1000 1003.

30. Nsonwu AC, Usoro CAO, Etykudo MH, Usoro IN. Influence of age, gender, and duration of diabetes on serum and urine levels of $\mathrm{Zn}, \mathrm{Mg}, \mathrm{Se}$ and $\mathrm{Cr}$ in type 2 diabetes in Calabar, Nigeria. Turk J Biochem 2006; 31(3):107-114.
31. Nasri $\mathrm{H}$. Lipids in association with serum $\mathrm{Mg}$ in diabetes mellitus patients. Acta Angiol 2006; 12(4):149-154.

32. Chhabra S, Chhabra S, Ramessur K, Chhabrs N. Hypomagnesemia and its implications in type 2 diabetes mellitus - A review article. Webmed Central Diabetes 2012; 3(12):WMC003878

33. Lopez-Ridaura R, Willett WC, Rimm EB, Liu S, Stamper MJ, Manson JE, Hu FB. Magnesium intake and risk of type 2 diabetes in men and women. Diabetes Care 2004;27(1): 134140.

34. Barbagallo $M$, Dominguez $L J$, Galioto $A$, et al. Role of magnesium in insulin action, diabetes and cardio-metabolic syndrome X. Molecular Aspects Medicine. 2003;24 : 39-52.

35. Vanquero MP. Magnesium and trace elements in the elderly, intake, status and recommendations. J Nutr Health Ageing 2002;6(2): 147-153

36. Kundu D, Osta M, Mandal T, Bandyopadhyay U, Roy D, Gautam D. Serum magnesium levels in patients with diabetic retinopathy. J Nat Sc Biol Med 2013; 4:113-116.

37. Mishra S, Padmanaban P, Deepti GN, Sarkar G, Sumathi S, Toora BD. Serum magnesium and dyslipidemia in type 2 diabetes mellitus. Biomed Res 2012;23(2): 295-300.

38. Hans CP, Sialy R, Bansal D. Magnesium deficiency and diabetes. Curr Sci 2002;83:1456-1463.

39. Sales $\mathrm{CH}$, Pedrosa LFC. Magnesium and diabetes mellitus: Their relation. Clinical Nutrition, 2006;25:554 - 562.

40. El-Yazigi AN, Hannan N, Raines DA. Urinary excretion of $\mathrm{Cr}_{\text {, }}$ $\mathrm{Cu}$, and $\mathrm{Mg}$ in diabetes mellitus and associated disorders. Diabet Res 1999;18:129-134.

41. Esfahani EN, Faridbod F, Larijani B, Ganjali MR, Norouzi $P$. Trace element analysis of hair, nail, serum and urine of diabetes mellitus patients by inductively coupled plasma atomic emission spectroscopy. Iran J Diab Lip Disor 2011;10: $1-9$.

42. Kisters K, Schildheuer M, Koneke J. Magnesium deficiency and increased fractional $\mathrm{Mg}$ excretion in insulin dependent diabetes mellitus, Mg loading test and blood pressure. Trace Elem Electrolytes 2000;17:67 - 70 .

43. Vanquero MP. Magnesium and trace elements in the elderly, intake, status and recommendations. J Nutr Health Ageing. 2002;6(2): 147-153.

44. Franz KB, Bailey SM. Geographical variations in hearth deaths and diabetes: effect of climate and a possible relationship to magnesium. J Am Coll Nutr 2004;23:521S-524S.

45. McNair P. Renal hypomagnesaemia in human diabetes mellitus, its relation to glucose homeostasis. Eur J Clin Invest. 1982; 112:81-85.

46. Swain R, Kaplan MB. Magnesium for the next millennium, Syndrome. Scan J Gastroenterol 1999;216(S):122-131.

47. Xu J, Xu W, Yao H, Sun W, Zhou W, Cai L. Associations of serum and urinary magnesium with the pre-diabetes, diabetes, and diabetic complications in the Chinese northeast population. PLOS ONE 2013; 8(2):1-8.

48. Song Y, Dai Q, He K. Magnesium intake, insulin resistance and type 2 diabetes. NAJ Med Sci 2013;6(1):9-15.

49. Singh $Y R$, Verma $S$, Agrawal $D$, Singh $B$, Bhardwaj A, Agrawal $G A$. A study of magnesium supplementation on glycemic control in patients of type-2 diabetes mellitus. Indian Journal of Clinical Anatomy and Physiology 2015;2(1):26-30.

50. Solati M, Ouspid E, Hosseini S, Soltani N, Keshavarz M, Dehghani M. Oral magnesium supplementation in type II diabetic patients. Med J Islam Repub Iran 2014;28:67-74. 